\title{
The judgment of distance on a plane surface
}

\author{
MICHAEL COOK \\ Australian National University, Canberra, A.C.T., Australia
}

\begin{abstract}
A test was made of the scalability of interval-ordering judgments of distance along the median path of a plane horizontal surface. Scales were derived from different subsets of judgments. It was found that scale values were related to distance by a power function with marked individual differences in exponent, but that different subsets of judgment yielded the same mean exponent. This finding was taken as positive evidence of scalability. The individual differences in judgment were shown to be reliable over a period of 3 weeks.
\end{abstract}

Many studies of visual distance perception have asked subjects to make quantitative judgments of distance. Such judgments have been treated in two ways. Quite frequently they have been reported directly. However, in a number of studies, they have been employed to construct judgmental scales. In constructing a scale, an experimenter selects a restricted class of distance judgment and attempts to represent the pattern of these judgments implicitly by means of some numerical function of physical distance. This is selected so that quantitative relationships between scale values embody judged distance relationships between the corresponding spatial locations. It is, of course, impossible to obtain judgments of all possible locations and, consequently, scales are constructed from subsets of distance judgments. The hope is that the scale might be used to predict judgments involving new locations not involved in the original scaling procedure. In practice, it will always be possible to construct a putative scale from judgments of an appropriately sampled subset of distances. However, there is no a priori guarantee of scalability in the sense that it would be possible to represent the complete set of potential distance judgments in the selected class by a single scale. This will only be possible if the pattern of judgment obeys certain formal constraints.

There has been no attempt to demonstrate empirically that distance judgments do possess the consistency necessary to enable them to be scaled. Nevertheless, scaling procedures have frequently been employed in the study of distance perception and there are many published perceived distance scales. Although a few studies have looked at judgments of suspended objects seen under various viewing conditions (Baird \& Biersdorf, 1967; Künnepas, 1960 Note 1), the majority have been concerned with judgments of location on a horizontal plane surface. Investigators have used a variety of procedures ranging

The author wishes to acknowledge the facilities provided during the preparation of this paper by the Department of Psychology, University of Edinburgh. from direct estimation of distances in physical units (Gibson \& Bergman, 1954; Gibson, Bergman, \& Purdy, 1955; Mershon, Kennedy, \& Falacara, 1977) through magnitude estimation (Baird \& Biersdorf, 1967; Galanter \& Galanter, 1973; Teghtsoonian \& Teghtsoonian, 1969, 1970; Künnepas, 1960, Note 1) and fractionation procedures (Gilinsky, 1951) to partition techniques (Gilinsky, 1951; Gogel, 1964; Rogers \& Gogel, 1975).

With a few exceptions (e.g., Gilinsky, 1951), the experiments have found scale values to be related to physical distance by a power function with a mean exponent in the vicinity of 1.0. However, even if we restrict interest to those studies which have examined judgments of distance on a horizontal plane, there are marked variations in the precise value reported for the mean distance exponent. Thus, Gogel (1964) and Teghtsoonian and Teghtsoonian (1969) report values less than unity, and this conclusion is implicit in data reported by Gilinsky (1951), Harway (1963), and Weckowicz, Sommer, and Hall (1958). On the other hand, Galanter and Galanter (1973) and Teghtsoonian and Teghtsoonian (1970) report mean exponents greater than unity, and the same result is implied by data reported by Gruber (1954), Newman (1971), Purdy and Gibson (1955), Wohlwill (1963, 1964), and Whitehouse and Gruber (Note 2). These inconsistencies are not surprising in the light of the heterogeneity in the conditions of the various experiments. Variations in mean exponent could be produced by differences in eye height (Wohlwill, 1963; but note the negative findings of Harway, 1963), instructions (Rogers \& Gogel, 1975), the range of distances tested (Teghtsoonian \& Teghtsoonian, 1970), the scaling task (Baird, 1970), or the difference between indoor and outdoor settings (Teghtsoonian \& Teghtsoonian, 1969, 1970), although no single one of these variables can account for all of the results cited. However, the question of the precise value of the mean exponent, or whether it is greater than or less than unity, is insignificant in the light of the observation of several studies that there are marked variations in the value of the distance ex- 
ponent for individual subjects, so that, under a given set of experimental conditions, some subjects yield an exponent less than unity and some yield a value greater than unity (Gogel, 1964; Mershon et al., 1977; Teghtsoonian \& Teghtsoonian, 1969).

This paper reports two experiments which provide further data concerning the form of the apparent distance scale. The main experiment (Experiment 1) was designed to establish whether distance judgments are, indeed, scalable. Experiment 2 was carried out to measure the reliability of the individual differences in judgment observed in Experiment 1. It was decided to restrict interest to interval-ordering judgments, in which one interval along the experimental path is to be judged as greater than, equal to, or less than another. These judgments can be given with relative confidence and reliability, and they avoid the problems which can arise if the subject is required to make numerical responses (Ekman, 1958; Wong, 1963). It was decided also to follow the majority of previous studies in examining the judgment of distances along the median path of a plane horizontal surface. This particular situation seems to offer a relatively good possibility of yielding a consistent pattern of judgment, since all potential locations on the experimental path are continuously visible to the subject. In requiring a judgment of a particular interval, then, the experiment is in a sense merely drawing the subject's attention to locations which are already visibly represented in the viewed scene.

Formally, complete representation of intervalordering judgments by a judgmental scale requires that it is possible to find a real function, $f(D)$, with the following property: that, for any four distances $D_{1}, \ldots, D_{4}$ along the experimental path, $\mid f\left(D_{1}\right)-$ $f\left(D_{2}\right)|<| f\left(D_{3}\right)-f\left(D_{4}\right) \mid$ implies that interval $\left(D_{1}\right.$, $\left.D_{2}\right)$ is judged less than interval $\left(D_{3}, D_{4}\right)$ and $\mid f\left(D_{1}\right)$ $-f\left(D_{2}\right)|=| f\left(D_{2}\right)-f\left(D_{4}\right) \mid$ implies that the two intervals are judged equal (or some stochastic equivalent of this property). It will only be possible to find such a function if distance judgments obey an appropriate set of constraints. We might attempt to specify these formally (see, for example, the axiomatizations of Blank, 1958, and Pfanzagl, 1968). However, such accounts are of necessity based upon highly idealized models of judgment and the assumptions involved are of a kind which makes them difficult to test empirically. Experiment 1 tested directly for the possibility of providing a metric representation of distance judgments by comparing a number of median distance scales constructed independently from different subsets of judgments. This strategy has commonly been adopted in testing the validity of scaling procedures (Chatterjea \& DasGupta, 1966; Guilford \& Dingman, 1954; Hanes, 1949).

\section{EXPERIMENT 1}

\section{Method}

Scaling procedure. Experimentally, the procedure was a version of the partition method employed by Gilinsky (1951), Gogel (1964) and Rogers and Gogel (1975). On the other hand, the scaling technique was a modification of the standard multiplication method (Stevens, 1957). In outline, the procedure was as follows:

The subject was positioned at the origin, $D=0$, of the median path. On each trial, an initial distance, $D_{1}$, was selected by the experimenter. Then a sequence of distances, $D_{2}, D_{3}, \ldots, D_{n}$ was determined, cutting off a succession of apparently equal intervals. That is, the intervals $\left(0, D_{1}\right),\left(D_{1}, D_{2}\right), \ldots,\left(D_{n-1}, D_{n}\right)$, were judged to be equal. $D_{1}$ was varied from trial to trial, with consequent variations in the distances $D_{2}, \ldots, D_{n}$. Let $m_{2}$ be the function relating $D_{2}$ to $D_{1}$, let $m_{3}$ be the function relating $D_{3}$ to $D_{1}$, and so on. Then the $i^{\text {th }}$ interval boundary was $D_{i}=$ $m_{i}\left(D_{1}\right)$. The functions $m_{2}, \ldots, m_{n}$ may be treated as equivalent, for analytic purposes, to conventional multiplication functions, so that $m_{2}$ may be described as a "doubling" function, $m_{3}$ as a "tripling" function, etc. However, the multiplication is implicit. The subject has been restricted to interval-ordering judgments and has not been required to estimate ratios.

Each of the multiplication functions $m_{2}, \ldots, m_{n}$ can be used independently to construct an estimate of the supposed median distance scale. If median distance judgments are, indeed, scalable, these $(n-1)$ estimates should all agree. In general, a scale can be constructed from a multiplication function by graphical procedures. However, if the multiplication functions have the linear form

$$
D_{i}=s_{i} D_{1}
$$

(the slope of the multiplication function, $s_{i}$, varying with $i$ ), this may be achieved algebraically (Stevens, 1957). It is necessary to select a unit by arbitrarily selecting a distance, $D_{0}$, and assigning it a scale value, $D_{0}^{\prime}$. Then, for the remaining points on the path, scale value, $D^{\prime}$ will be related to distance by the power function,

$$
\mathbf{D}^{\prime}=\mathbf{k D}^{\beta} \text {. }
$$

The exponent of this function is related to the slope, $s_{i}$, of the $i^{\text {th }}$ multiplication function by

$$
\beta=\log i / \log s_{i},
$$

(Stevens, 1957) and the multiplicative constant, $k$, is given by

$$
k=D_{o}^{\prime} D_{o}^{-\beta} \text {. }
$$

$\mathrm{k}$ is a dimensional constant only and is of no empirical significance. This means that in order for the distance scales constructed from the different multiplication functions to agree, it is necessary only that each of the latter should yield the same value of $\beta$. These can be estimated from the slopes of the multiplication functions using Equation 2.

Apparatus and Procedure. No attempt was made to restrict viewing. Subjects had a clear view of the edges of the experimental surface, the laboratory, and the experimenter. The laboratory measured $15 \times 12 \mathrm{~m}$. Tables and chairs were piled around the walls, but all objects were removed to a distance of at least $3 \mathrm{~m}$ from the experimental surface. This was a horizontal plane rectangle measuring $540 \times 150 \mathrm{~cm}$. It was covered by a fine grid pattern (a gray-and-white gingham cloth with a 1-cm cycle), which was arranged so that the grid lines were orthogonal to the edges of the experimental surface. The longitudinal lines of the pattern were visible over the whole surface length, but the transverse lines were not discriminable in its further half. The sub- 
ject was seated so that he viewed the surface from a point perpendicularly above its shorter edge and so that his median plane bisected the surface in the longer dimension. He was required to maintain this position as nearly as possible, but was allowed to rotate his head freely while making judgments. The mean eye height was $36.3 \mathrm{~cm}$, with a standard deviation of $5.8 \mathrm{~cm}$. Viewing was binocular.

Estimates were made of the five multiplication functions $m_{2}$, $\ldots, m_{6}$. Positions on the surface were marked by six $1-\mathrm{cm}$ steel rods, varying randomly in length between 88 and $113 \mathrm{~cm}$, which were laid perpendicularly across the median path. The experimenter adjusted the positions of these markers by rolling them along the surface. Settings were obtained for $D_{1}=25,38,50$, $75,100,125,150$, and $175 \mathrm{~cm}$. On any trial, an initial standard rod was placed across the median path at the selected $D_{1}$. A second variable rod was then placed across the path, and the subject was asked to compare the distance separating the initial rod from the surface edge with the distance between the two rods. The subject was instructed to judge on the basis of apparent physical distance. That is, the far interval was to be judged, say, less than the near one if the tape-measure distance along the surface looked smaller. The position of the far rod was adjusted by the twocategory method of limits until a change of judgment occurred. From this position, it was moved back half a step $(0.75 \mathrm{~cm})$ towards the starting point of the sequence. This position was taken as an estimate of $D_{2}=m_{2}\left(D_{1}\right)$. A third rod was then placed on the surface, and its position was adjusted until the three visible intervals appeared equal. This procedure was repeated until, where possible, six rods lay on the surface. However, the limitation in the length of the experimental surface meant that it was not always possible to obtain settings when both $i$ and $D$ were large. Two trials were given for each of the eight values of $D_{1}$. Within any trial, ascending and descending sequences were employed at random in adjusting the successive rods, with the constraint that over the whole experiment, for any $i$ and $D_{1}, m_{i}\left(D_{1}\right)$ was estimated once by an ascending and once by a descending series.

Subjects. Twenty subjects took part in the experiment, comprising 11 females and 9 males. Five had corrected vision. Eighteen were student volunteers, participating to earn course credit. Two were postgraduate students in psychology.

\section{Results}

Figure 1 shows the mean forms (averaging over individuals) of the five multiplication functions estimated in this experiment. In calculating each of these, it was necessary, in view of the incomplete nature of the data, to make a selection of both the subjects and the range of values of $D_{1}$ to be included, so that each subject contributed to every point in a given mean function. The figure indicates the number of subjects included in each case.

Visually, the illustrated functions approximate closely to the linear form of Equation 1. This was tested statistically in two ways. In the first place, analyses of variance were performed upon the illustrated functions. None of these yielded a significant deviation from linearity. In the second place, straight lines were fitted by least squares to each multiplication function for every individual. For no function was the mean intercept (averaging over individuals) significantly different from zero. It was therefore assumed that Equation 1 held for each individual and multiplication function. Accordingly, the coefficient $s_{i}$ in this equation was estimated individually for each case as the slope of the best fitting straight

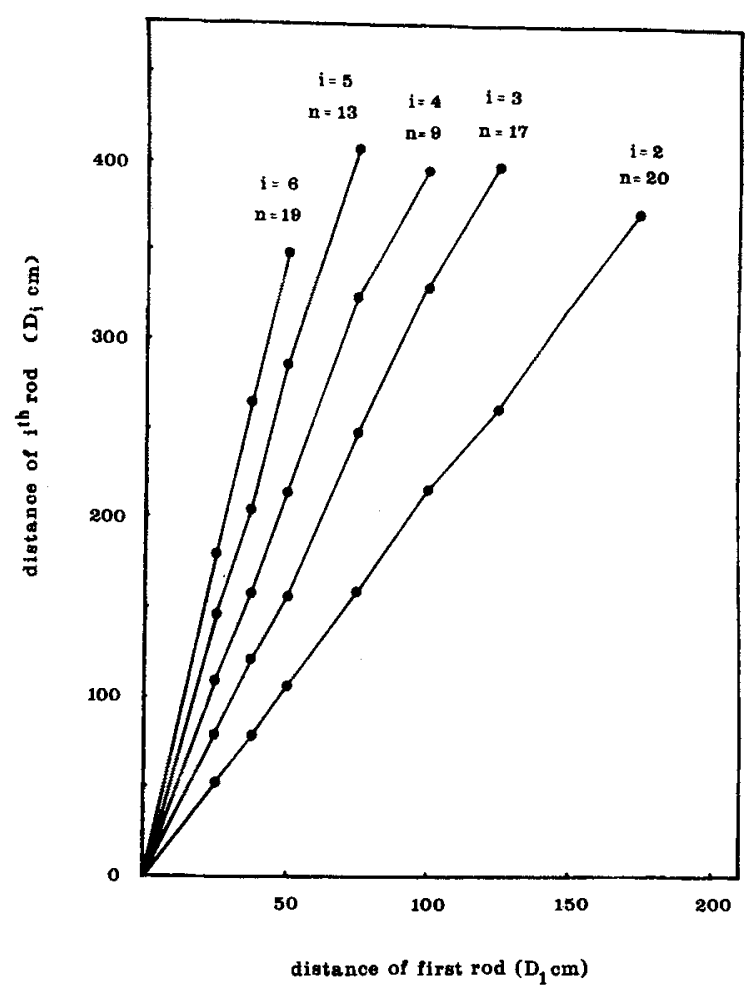
$\mathbf{D}_{1}$.

Figure 1. Mean multiplication functions: $D_{i}$ as a function of

line fitted to the variable settings by least squares so as to pass through the origin (this time using all available measurements). Note that, provided the linearity of the multiplication functions continued beyond the experimental range of $D$, the missing data should have had no systematic effect upon the estimate of $\mathrm{s}_{\mathrm{i}}$. Teghtsoonian and Teghtsoonian (1969), in fact, found no effect of range on distance judgments made in an indoor setting. But, in any case, this procedure meant that although the individual multiplication functions were based upon different numbers of points, they were all determined within the same range of $\mathrm{D}$. This was appropriate, since we wished to determine the consistency of judgment within a given range.

If a given multiplication function has the form specified by Equation 1, the judgmental scale constructed from this function will be related to median distance by Equation 2 and the exponent $\beta$ in this equation may be estimated by Equation 3. This constant was estimated separately for each individual and multiplication function. Averaging over the five estimates of $\beta$ for each individual yielded a best estimate of the constant for that person. The range of $\beta$ in this sample was 0.776 to 1.183 , with a mean of 0.927 and a standard deviation of 0.079 . This mean is significantly less than $1.0, \mathrm{t}(19)=4.13, \mathrm{p}<.01$.

If median distance judgments do have the consistency required to make them scalable, all multiplication functions for a given subject should yield the 
same value of $\beta$. Figure 2 shows the mean value of $\beta$ (averaging over individuals) for each multiplication function ( $i=2, \ldots, 6)$, together with the values for the two extreme individuals. No systematic differences are evident between the mean exponents yielded by the different multiplication functions. This was confirmed by an analysis of variance (two-way mixed model with one entry per cell), which yielded no significant main effect for multiplication functions, $F(4,76)=1.53, p<.05$, and a highly significant effect for subjects, $F(19,76)=47.66, p<.001$. While this analysis did not permit a test for a Subjects by Functions interaction, the raw data gives no indication of any systematic effect of this sort and any interaction must, at most, have been small, since the residual SS was only $7 \%$ of the total SS.

\section{EXPERIMENT 2}

The highly significant main effect for subjects observed in Experiment 1 provides strong evidence of individual differences in distance judgment. However, the differences there manifested themselves only within a single experimental session. The second experiment was carried out to determine whether they would be maintained across experimental sessions.

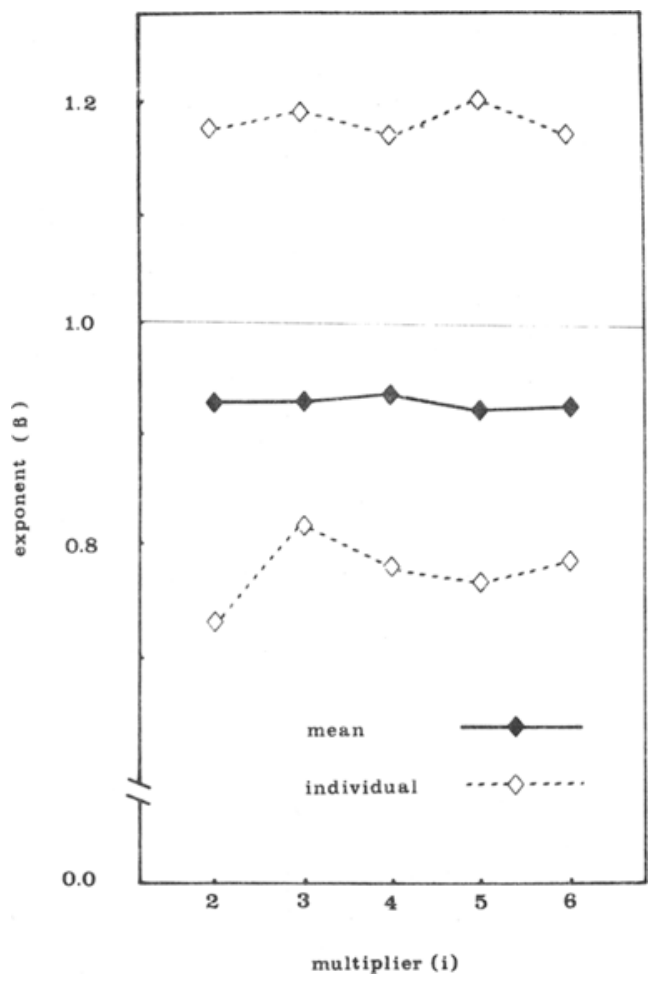

Figure 2. Average and extreme individual estimates of the exponent of the median distance scale for different multiplication functions.

\section{Method}

Scaling method. The scaling procedure differed from that employed in Experiment 1, being a version of the standard bisection method (Stevens, 1957). On any trial, the experimenter nominated a position, $D_{e}$, on the experimental path and the subject was required to divide the interval $\left(0, D_{e}\right)$ into two apparently equal subintervals. The bisection function, which relates the bisecting distance, $D_{b}$, to $D_{e}$ was determined individually for each subject. The procedure for constructing a scale from this function then corresponded closely to that described for Experiment 1 . If the form of the distance scale is a power function, the bisection function will have the linear from, $D_{b}=s_{b} D_{e}$, and the exponent of the distance scale may be calculated from the slope, $s_{b}$, of the bisection function using Equation 2, which, in this case, becomes $\beta=\log (0.5) / \log s_{b}$.

Apparatus and Procedure. The experiment employed the same experimental surface and viewing conditions as Experiment 1. Locations on the surface were marked by two rods which were selected at random on each trial from the pool of six rods used in the earlier experiment. As before, the subjects were asked to compare the apparent tape-measure distances between the interval boundaries. On any trial, the far rod was placed at a selected distance and the experimenter then adjusted the position of the nearer rod using a two-category method of limits. One ascending and one descending sequence was given for $D_{e}=51,102,163$, 254,381 and $508 \mathrm{~cm}$. The order of the 12 trials was randomized for each subject. Each subject was retested at the same time on the following day and again after an interval of 21 days.

Subjects. Twenty-one subjects took part in the experiment, comprising 9 males and 12 females. All were student volunteers, participating to earn course credit. None had taken part in Experiment 1 . Seven had corrected vision.

\section{Results}

The mean bisection function obtained in each session was tested for linearity using the procedures described for Experiment 1. No significant nonlinearity was found. Accordingly, an estimate of the exponent $\beta$ was made individually for each subject on each experimental session. Each estimate was calculated from the least-squares estimate of the slope of the bisection function for that session. This procedure yielded, for the first session, a distribution of 21 estimates of $\beta$ with a mean of 0.966 , a standard deviation of 0.100 and a range between 0.777 and 1.299. Unlike that obtained in Experiment 1, this mean did not differ significantly from unity, $t(20)$ $=1.56, \mathrm{p}>.05$. However, the means obtained in the respective experiments did not differ significantly from each other, $t(39)=1.48, p>.05$. The productmoment correlation between the pairs of estimates of the distance exponent obtained in the first and second sessions was 0.891 and the correlation between those obtained in the first and third sessions was 0.883 .

\section{DISCUSSION}

The most important finding of this study is the identity of the five different estimates of $\beta$ obtained in Experiment 1 . In effect, this means that a subject's placement of the five variable rods $\left(D_{2}, \ldots\right.$, $\left.D_{6}\right)$ for any given position of the standard $\left(D_{1}\right)$ was 
metrically consistent with the way in which the placement of any one variable altered with the position of the standard. Given the fact that this was combined with errors in judgment which were quite substantial for some subjects, this is quite a strong test of consistency and, hence, provides a reasonably strong confirmation of the scalability of distance judgments within the experimental range of D. One argument which might be raised against this conclusion is that the consistency of judgment was artificially raised by the experimental conditions, in particular by cues provided by the unrestricted view of the experimenter and the surrounding laboratory. However, this argument would have to account for the fact that consistency was achieved in the face of systematic errors in judgment. It is difficult to see how this combination of internal coherence and systematic error could have been maintained if the subjects were basing their judgments upon contextual cues, since any extraneous cue which helped the consistency of judgments would be expected at the same time to yield objectively correct judgments.

The finding that the form of the distance scale is a power function confirms the results of numerous earlier studies. Under the conditions of the present study, the mean exponent of this function was slightly less than 1.0 (significantly so only in the case of Experiment 1). However, as was pointed out earlier, the precise value of this mean has been shown to be influenced by many variables and the particular value obtained here is probably specific to the conditions of this study. Of greater interest is the confirmation of earlier reports of individual differences in judgment. The results of Experiment 1 showed that these differences are reliable within an experimental session, and Experiment 2 demonstrated that they are maintained over a period as long as 3 weeks. If we pool the results of the two experiments, we obtain a distribution for a total of 41 individuals. Values in this distribution range from 0.776 to 1.229 with a mean of 0.947 and a standard deviation of 0.090 . This mean is significantly less than $1.0, \mathrm{t}(40)=3.77, \mathrm{p}<.01$. [It is worth noting that this distribution is very similar to that obtained by Mershon et al. (1977) under somewhat different experimental conditions and for direct estimates of distance in physical units.] The interesting feature of this distribution is the fact that the range of $\beta$ included unity. In fact, $\beta$ was greater than 1.0 for approximately $25 \%$ of the pooled sample.

The difference in behavior between those for whom $\beta<1.0$ and those for whom $\beta>1.0$ is striking, and it is tempting to regard these as two distinct groups of subjects who display some significant difference in the process of judgment. However, the frequency distribution of exponents in the pooled sample was symmetrical and unimodal and displayed no perturbation in the region of $\beta=1.0$.
Furthermore, if we consider the subject's behavior in proximal rather than distal terms, a continuity of behavior becomes evident. On any trial of the multiplication or bisection methods, the subject is to provide a sequence of positions along the median path cutting off apparently equal intervals. If these intervals are to be equal, it is necessary that the retinal projection of each be smaller than that of its predecessor. The task for the subject's perceptual system is to determine how much smaller. The results of these experiments show that all subjects behaved in an appropriate way in providing a sequence of intervals subtending progressively smaller angles and that the rate of decrease was approximately of the order required (this may be seen by plotting scale values against angular rather than distance coordinates). However, it seems that for some subjects the rate of angular decrease was insufficient, yielding an apparent "expansion" of the plane and a value of $\beta$ greater than unity, while for the majority the rate was too great, yelding a value of $\beta$ less than unity and an apparent "compression" of the plane.

It still remains necessary, however, to explain why subjects differed in their distance judgments. Correlations were calculated (for the pooled sample) between the distance exponent and various extraneous subject-variables that might have contributed to the differences between individuals, namely, experimental eye height, standing eye height, sex, age, and correction/noncorrection of vision. However, in each case, the correlation was small and statistically insignificant. It seems likely, therefore, that the observed variations arose from differences in the actual process of judging distance. One possible source of such differences lies in the suggestion (Gogel, 1973; Rogers \& Gogel, 1975) that, in judging distances, people deliberately adjust their judgments to make allowances for supposed tendencies towards underconstancy. This would account for the present findings if we also assume that there were individual differences in the correlations employed. Mershon et al. (1977) offer precisely this explanation of the individual differences in distance judgment that they observed, citing, in support their finding, that the differences are reduced (though not eliminated) by the use of calibration equations based upon motor responses. Of course, this explanation still leaves unanswered the question of why there should be variations in the corrections employed by individual subjects, but it does remove the explanation of these differences from the purely perceptual domain. The data from this study neither affirm nor deny this account. However, they do imply that the corrections, if they are made, are of a sophisticated nature, being sufficiently subtle to maintain the coherent pattern of judgment observed in Experiment 1 and sufficiently stable to yield the reliability observed in Experiment 2. 


\section{REFERENCE NOTES}

1. Künnepas. T. Distance perception as a function of available cues (Report No. 231). Stockholm: Psychological Laboratory, University of Stockholm. 1967.

2. Whitehouse, J. M., \& Gruber, H. E. The effects of surface texture and binocular disparity on distance-ratio perception. Paper read to the Colorado Psychological Association, Fort Collins, 1957.

\section{REFERENCES}

BAIRD, J. C., \& BIERsDorf. W. R. Quantitative functions for size and distance judgments. Perception \& Psychophysics, 1967, 2, $161-166$

BaIro, J. C. Psychophysical analysis of visual space. Oxford: Pergamon, 1970.

BIANK, A. A. Axiomatics of binocular vision. Journal of the Optical Society of America, 1958, 48, 328-334.

Chatterjea, R. S., \& DasGupta, B. Scale of weight from fractionation and multiplication data. A merican Joumal of Psychologv. 1966, 79, 116-119.

EKMAN, G. Two generalized ratio scaling methods. Journal of Psychology, 1958, 45, 287-295.

Galanter, E., \& GALANTER, P. Range estimates of distant visual stimuli. Perception \& Psychophysics, 1973, 14, 301-306.

Gibson. E. J.. \& Bergman. R. The effect of training on absolute estimation of distances over ground. Journal of Experimental Psychology. 1954. 48, 473.482.

Gibson. E. J., Bergman, R., \& Purdy, J. The effect of prior training with a scale of distance on absolute and relative judgments of distance over ground. Journal of Experimental Psychology, 1955, 50. 97-105.

Gilinsky, A. S. Perceived size and distance in visual space. Psychological Review, 1951, 58, 460-482.

GoGEL, W. C. Visual perception of spatial extent. Journal of the Optical Society of America, 1964, 54, 411-416.

GoGel, W. C. The organization of perceived space: II. Consequences of perceptual interactions. Psychologische Forschung, 1973. 36. 223-247.

GRUBER, H. E. The relation of perceived size to perceived distance in visual space. American Journal of Psychology, 1954, 67, 411-426.

Guilford, J. B., \& Dingman, H. F. A validation study of ratiojudgment methods. American Journal of Psychology, 1954, 67, 395-410.
Hanes, R. M. The construction of brightness scales from fractionation data: A validation. Joumal of Experimental Psychology, 1949, 39, $719-728$.

HARWAY, N. I. Judgment of distance in children and adults. Journal of Experimental Psychology, 1963, 65, 385-390.

KüNNEPAS, T. Scales for subjective distance. Scandinavian Journal of Psychology, 1960, 1, 187-192.

Mershon, D. H., Kennedy, M., \& Falacara, G. On the use of "calibration equations" in perception research. Perception, 1977 , 6. 299-311.

Newman, C. V. The influence of visual texture density gradients on relative distance judgments. Quarterly Joumal of Experimental Psychology, 1971, 23, 225-233.

Pfanzagl, J. Theory of measurement. New York: Wiley, 1968.

Purdy, J., \& Gibson. E. J. Distance judgment by the method of fractionation. Journal of Experimental Psychology. 1955, 50. 374-380.

Rogers, S. P., \& Gogel. W. C. The relation between judged and physical distance in multicue conditions as a function of instruc. tions and tasks. Perceptual and Motor Skills, 1975, 41, 171-178.

Stevens, S. S. On the psychophysical law. Psychological Review, 1957, 64, 153-181.

Teghtsoonian, M., \& Teghtsoonian, R. Scaling apparent distance in a natural outdoor setting. Psychonomic Science, 1970, 21, 215-216.

WECkowicz, T. E., SOMmer, R., \& HALl, R. Distance constancy in schizophrenic patients. Joumal of Mental Science, 1958, 104, 1174-1182.

Weckowicz, T. E., Sommer, R., \& Hall, R. Distance constancy in schizophrenic patients. Joumal of Mental Science, 1958, 104. 1174-82.

WoHLwill, J. F. Overconstancy in distance perception as a function of the texture of the stimulus field and other variables. Per. ceptual and Motor Skills, 1963, 17, 831-846.

WoHLwill, J. F. Changes in distance judgments as a function of corrected and uncorrected practice. Perceptual and Motor Skills, 1964, 19, 403-413.

WONG, R. Effect of the modulus on estimates of magnitude of linear extent. American Journal of Psychology, 1963, 76, 511-512.

(Received for publication August 24, 1977; revision accepted September 19, 1977.) 